\title{
Stress generation during the quenching of large AA2618 forgings: Finite element computations and validation against neutron diffraction measurements
}

\author{
N. Chobaut ${ }^{\mathrm{a}}$, J.N. Wagner ${ }^{\mathrm{b}}$, D. Carron ${ }^{\mathrm{c}}$, P. Saelzle ${ }^{\mathrm{d}}$, T. Pirling ${ }^{\mathrm{e}}$, M. Hofmann ${ }^{\mathrm{f}}$, J.-M. Drezet $^{\mathrm{a}, *}$ \\ a Ecole Polytechnique Fédérale de Lausanne, Laboratoire de Simulation des Matériaux, station 12, 1015 Lausanne, Switzerland \\ b Paul Scherrer Institut, Materials Science and Simulation, ASQ/NUM, 5232 Villigen PSI, Switzerland \\ ${ }^{\mathrm{c}}$ Univ. Bretagne Sud, FRE CNRS 3744, IRDL, F-56100 Lorient, France \\ d ABB Turbo Systems Ltd, Bruggerstrasse 71A, 5401 Baden, Switzerland \\ e Institut Laue-Langevin, 6 rue Jules Horowitz, 38042 Grenoble, France \\ ${ }^{\mathrm{f}}$ Forschungsneutronenquelle Heinz Maier-Leibnitz (FRM II), TU München, Lichtenbergstr. 1, 85747 Garching, Germany
}

\section{A R T I C L E I N F O}

\section{Keywords:}

Aluminium alloys

Quenching

Residual stress

Neutron diffraction

Strain gauges

\begin{abstract}
A B S T R A C T
Solutionising and quenching are key steps in the fabrication of heat treatable aluminium parts such as AA2618 compressor impellers for turbochargers. Quenching not only dictates the mechanical characteristics of the product but also induces residual stresses that can cause unacceptable distortions during machining and unfavourable stresses in service. Predicting and controlling stress generation during quenching of large AA2618 forgings is therefore of great interest. Since possible precipitation during quenching may affect the local yield strength of the material and thus impact the level of macro-scale residual stresses, consideration of this phenomenon is required.

A phenomenological material model accounting for precipitation in a simple way is used instead of modelling in detail precipitation that occurs during quenching. The required model parameters are identified using a limited number of tensile tests achieved after representative interrupted cooling paths in a Gleeble machine. This model is used in FE computations of stress generation during quenching of large massive AA2618 forgings for compressor impellers. The residual strain and stress profiles are compared with neutron diffraction measurements carried out at SALSA and STRESS-SPEC diffractometers in as-quenched and in T6 conditions. It turned out that the residual stress predictions by FE modelling might be wrong if precipitation is not taken into account properly in the material model.
\end{abstract}

\section{Introduction}

In heat-treatable aluminium alloys (AA), quenching from the solutionising temperature is of prime importance to control the final properties of the part. From a metallurgical viewpoint, the ideal quench must be fast enough to avoid the formation of precipitates during quenching, i.e. to obtain a supersaturated solid solution. However, ideal fast quenching cannot be achieved in the centre of large massive components where the quenching rate can be more than ten times lower than at the surface. This leads to the possible formation of coarse precipitates which are detrimental to the final yield strength. Indeed, coarse precipitates decrease the hardening potential by pumping solute atoms during quenching and influence the as-quenched residual stresses (RS) that depend also on the magnitude of the cooling rates and on the component size and shape.
The Al-Cu-Mg-based AA2618 alloy is a wrought alloy that is widely used for the compressor impellers in turbochargers for combustion engines owing to its high creep resistance [1]. The impellers are typically machined directly out of a forged blank, which has undergone a T6-heat treatment involving solutionising, quenching and artificial ageing. In order to reduce residual stresses, quenching is performed in boiling-water rather than in cold-water. In the artificial ageing step only a modest reduction in stresses is expected [2].

The prediction of internal stress build-up during quenching involves the determination of the transient heat transfer in the part and requires an adequate material model for finite element (FE) calculations accounting for the appropriate material properties.

A material model, based on interrupted quench tests in a Gleeble machine and validated upon constrained cooling (blocked-jaws) tests, has been developed by the authors [3]. In this model the impact of

\footnotetext{
* Corresponding author

E-mail address: jean-marie.drezet@epfl.ch (J.-M. Drezet).
} 


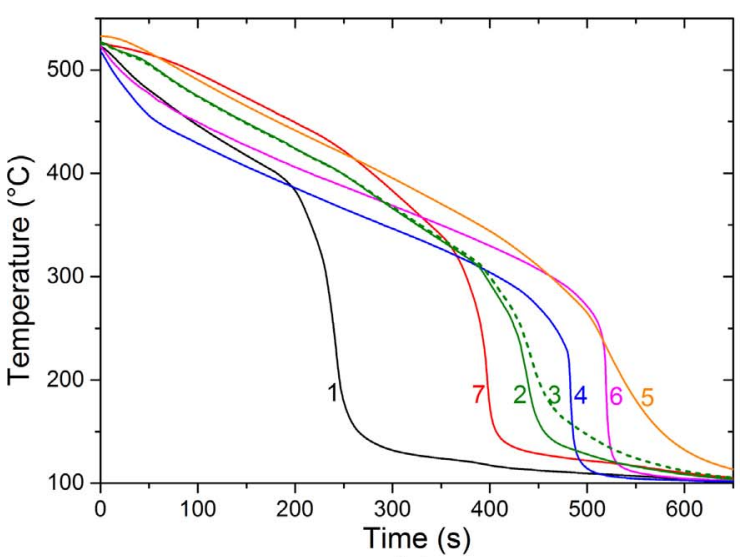

(a)

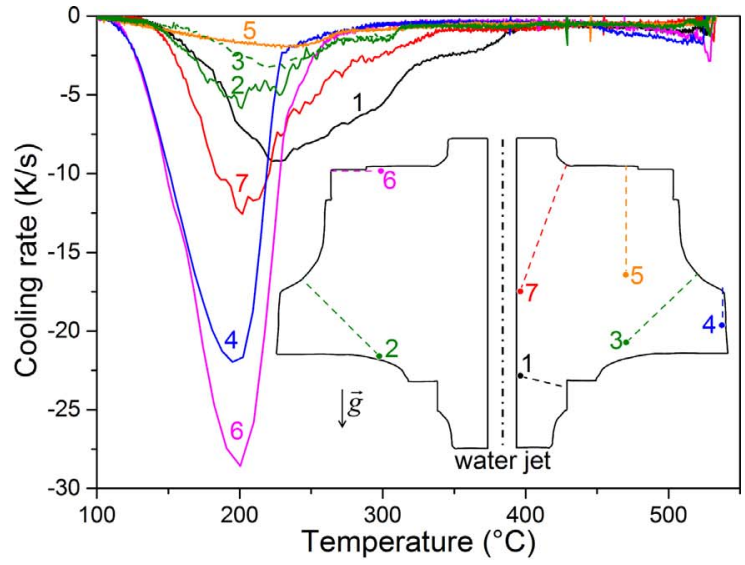

(b)

Fig. 1. (a) Measured cooling curves in boiling-water quenched forging A and (b) corresponding cooling rates. Inset: position of thermocouples with drillings in dashed lines.

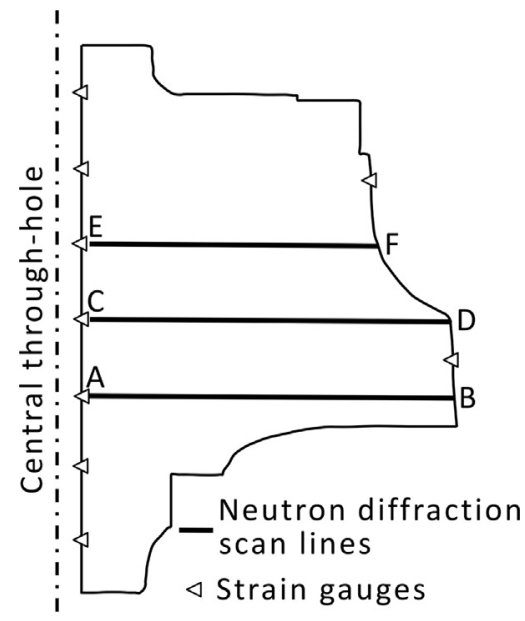

Fig. 2. Positions of rosette strain gauges and neutron diffraction scan lines $[\mathrm{AB}],[\mathrm{CD}]$ and $[\mathrm{EF}]$.

precipitation during cooling is considered through temperature dependent yield strength thus avoiding a complex and fastidious characterisation of precipitation during quenching. The material model is now applied to quenching simulations of large impeller forgings which experience unconstrained cooling. This is more complex than uniaxial blocked-jaws tests as it requires an accurate knowledge of the complex

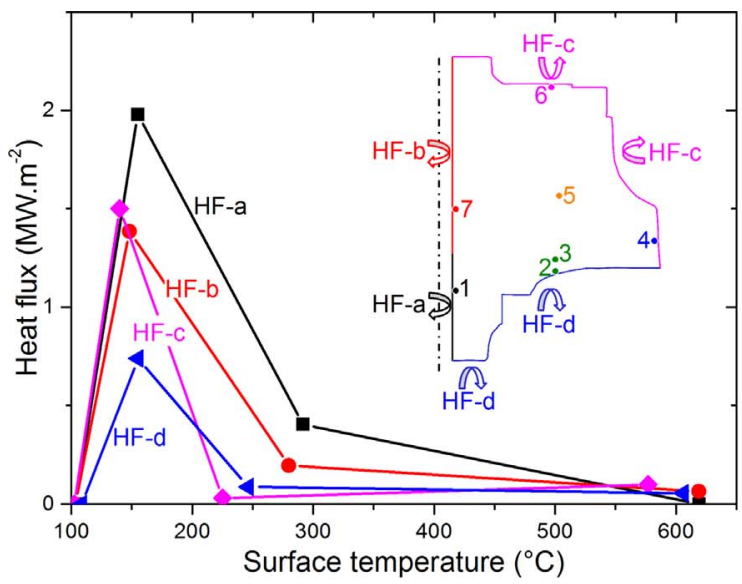

(a) transient thermal field for stress-strain FE simulations.

Temperature measurements during the entire heat treatment followed by RS measurements in T6 state using neutron diffraction (ND) and subsequent dissection method with strain gauges have been executed on one forging with central hole. Temperature measurements were used in an inverse modelling to calculate the position and temperature dependent heat fluxes (HFs). On a second forging with central hole of identical geometry and with the same heat treatment, RS measurements by ND were conducted in as-quenched and then in T6 states in order to quantify the amount of relaxation during artificial ageing and to check the reproducibility of the quench on both forgings. Based on the determined HFs and the validated material model, the stress generation during quenching has been simulated by FEA for the given forging with hole, and the therewith predicted as-quenched strains and stresses are compared to the RS measurements. Finally the results are compared to similar calculations performed on a forging of almost identical outer geometry but without a central axial throughhole, which decelerates the cooling speed in the center [4], in order to discuss the effect of cooling on precipitation and residual stress.

\section{Experimental procedure}

Two identical axisymmetric AA2618 forgings of about $0.5 \mathrm{~m}$ in diameter, with a central axial through-hole of $6 \%$ diameter of the outer diameter, and a total weight of about $110 \mathrm{~kg}$ were used:

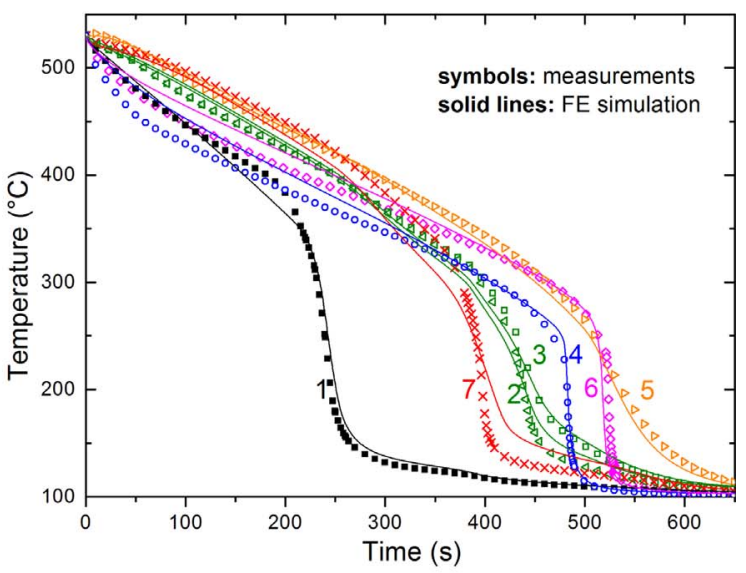

(b)

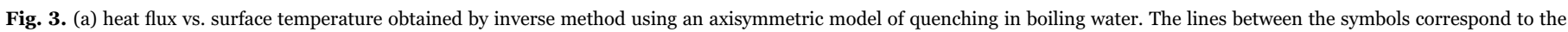
linear interpolation performed in-between the optimised heat fluxes in the FE simulation. (b) comparison between temperature measurements and simulation. 


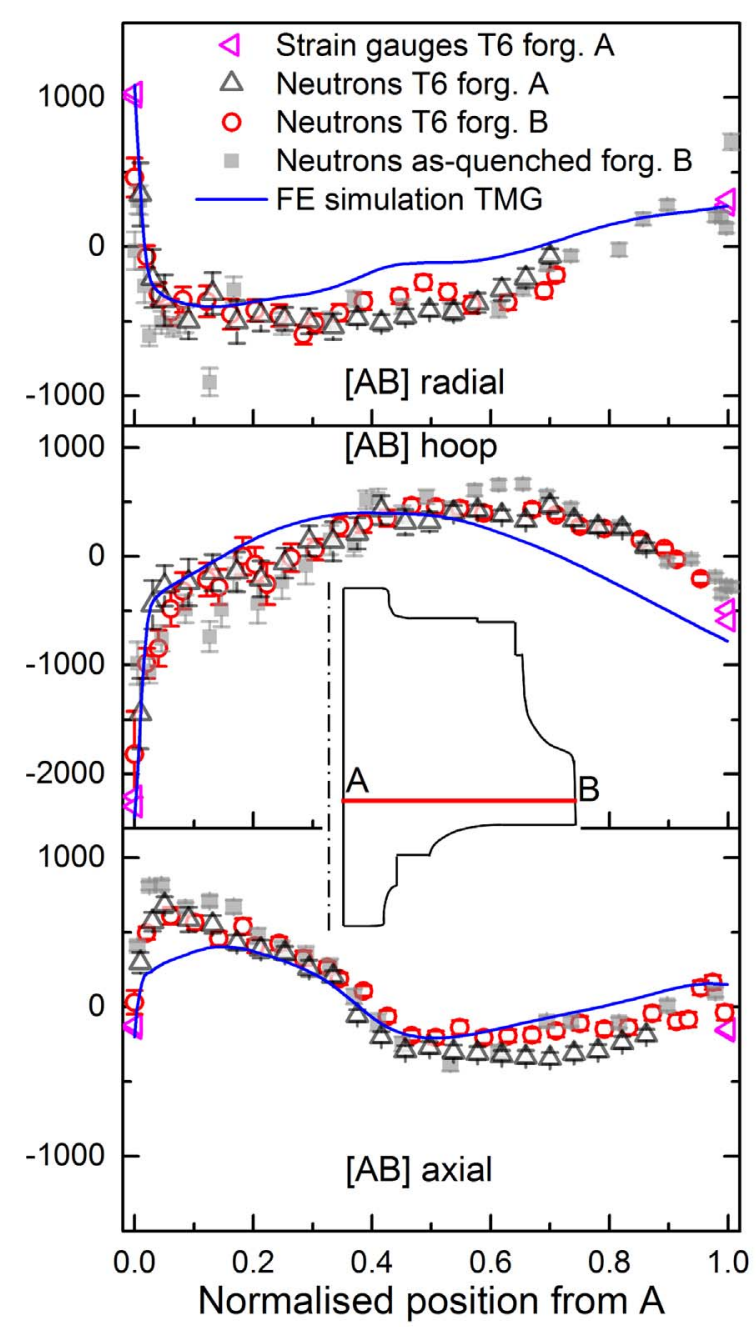

(a)

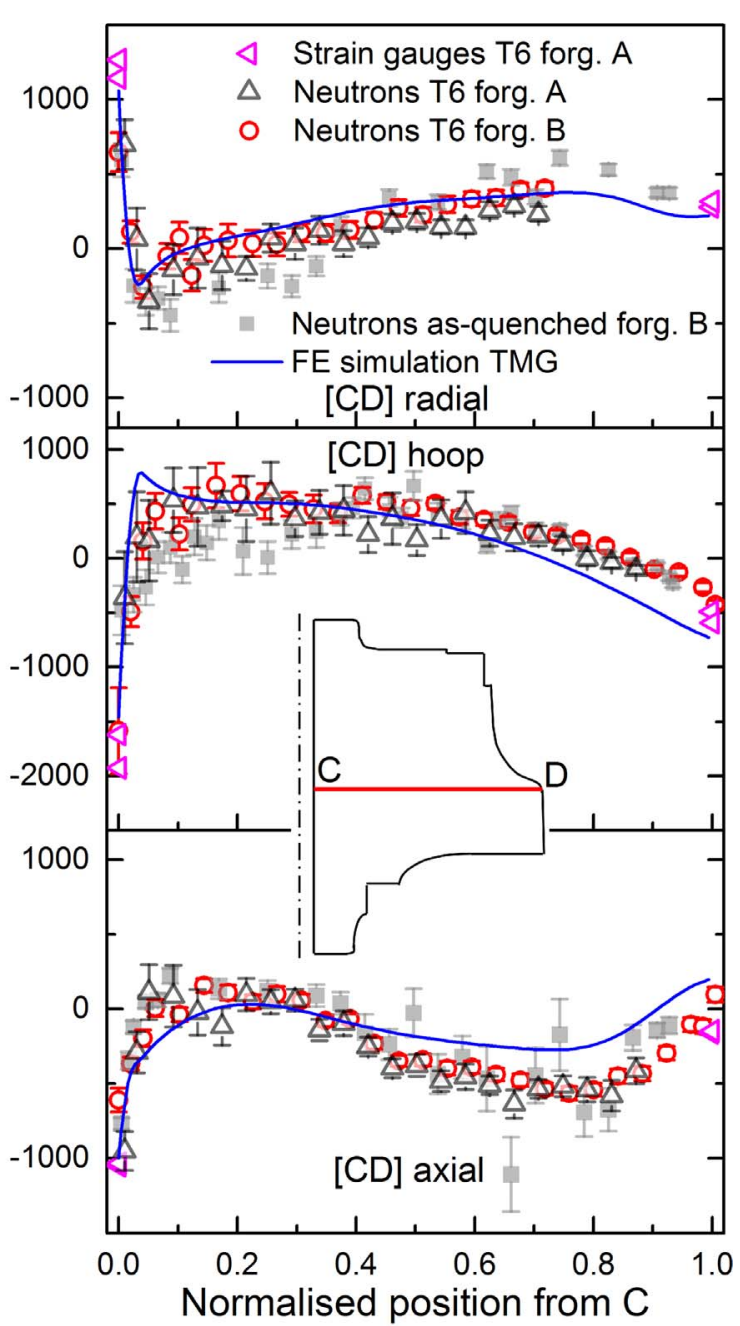

(b)

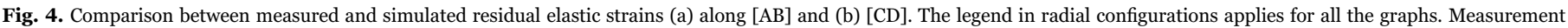
data refer to as-quenched and $\mathrm{T} 6$ condition, simulation consider the as-quenched condition.

- Forging A: temperature measurements followed by RS measurements in T6 state by ND and subsequently by dissection method with strain gauges,

- Forging B: RS measurements by ND in as-quenched and in T6 state.

To determine the heat transfer during quenching, forging $\mathrm{A}$ has been equipped with 7 type-K thermocouples (TCs) $1.5 \mathrm{~mm}$ in diameter, some of them located only $3 \mathrm{~mm}$ beneath the surface. In T6 state, i.e. after quenching and ageing for $24 \mathrm{~h}$ at $473 \mathrm{~K}\left(200{ }^{\circ} \mathrm{C}\right)$, the same forging was taken for RS measurements by ND and subsequently equipped with 14 rosette strain gauges for strain measurements with dissection method.

Forging $\mathrm{B}$, which has experienced the identical heat treatment, was non-destructively tested by ND, first in the as-quenched state and then in T6 state. These measurements allow quantifying the amount of relaxation during artificial ageing and checking the reproducibility of the quench.

The heat treatment of both forgings has been carried out individually in an industrial heat treatment installation.

\subsection{Temperature measurements during quenching}

After solutionising the forgings have been moved within less than $30 \mathrm{~s}$ from the furnace to the quenching basin with boiling water and immersed vertically. In the basin, a lance ejects water through the axis of the forgings in order to increase the cooling rate in the temperature range of $673-573 \mathrm{~K}\left(400-300{ }^{\circ} \mathrm{C}\right)$ by avoiding extensive film boiling regime. Thus it also reduces the amount of large precipitates that might form in this temperature range.

Thermal measurements were performed with a sampling rate of $3 \mathrm{~Hz}$ using the precision NetDAQ Networked Data Acquisition Unit together with the NetDAQ Logger software.

From the solutionising temperature, temperature begins to decrease almost linearly with time but is not uniform within the forging as shown in Fig. 1.

The bore and the bottom side, which are exposed to the water jet, experience the fastest cooling. From $573-673 \mathrm{~K}\left(300-400^{\circ} \mathrm{C}\right)$ on, depending on their position, the TCs close to the surface show a sharp temperature drop due to the transition from film boiling to nucleate boiling regime. The slowest cooling is registered by TC5 which is close to the centre of the massive cross section. The overall inhomogeneity of the cooling within the forging results in huge temperature gradients inducing internal thermal stresses. The inhomogeneity is very pronounced in the bore where the temperature drop at TC1 occurs about $2 \mathrm{~min}$ before the drop at TC7, which is only about $10 \mathrm{~cm}$ apart. This is due to the fact that the transition regime in the bore is concentrated over a small area and moves slowly from the bottom to the upper region. This phenomenon has been investigated experimentally and numerically by Büche et al. [5].

As temperature approaches $373 \mathrm{~K}\left(100{ }^{\circ} \mathrm{C}\right)$, i.e. the quenchant 


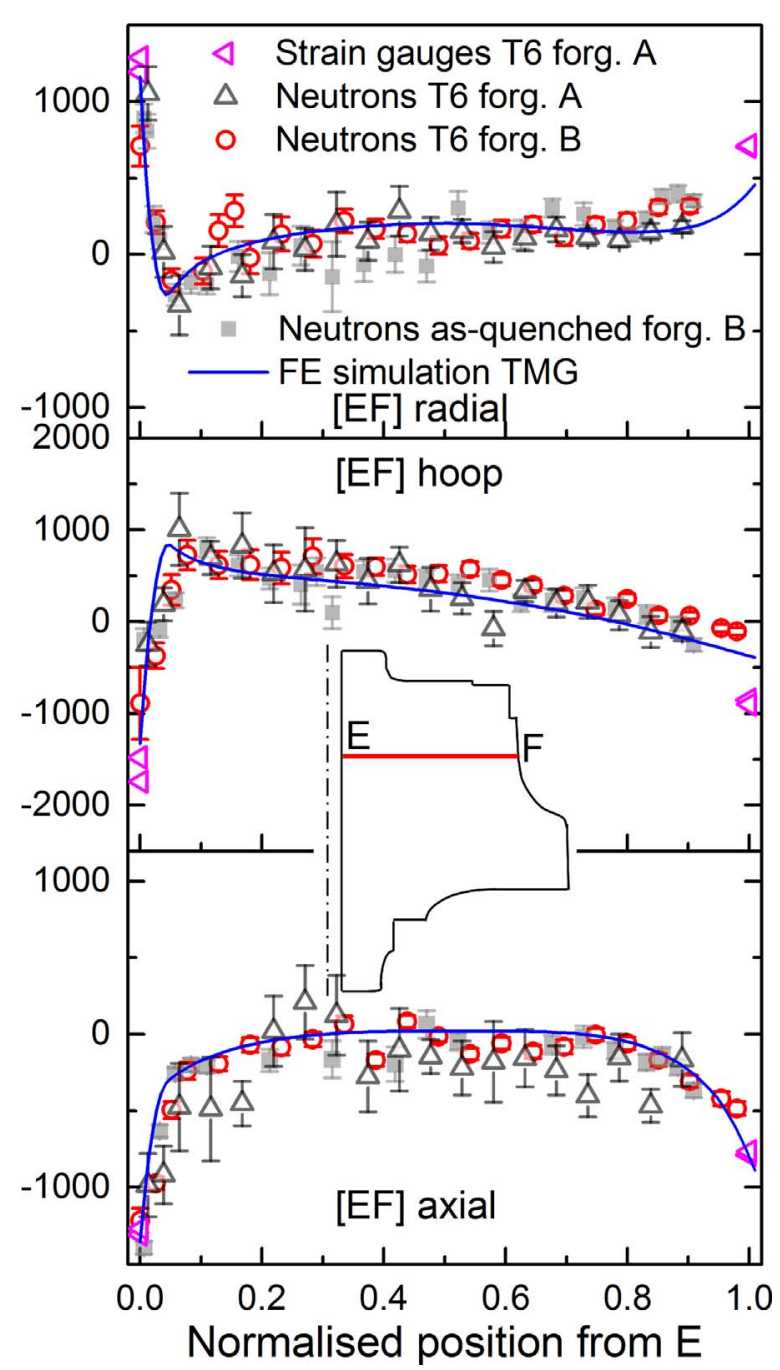

Fig. 5. Comparison between measured and simulated residual elastic strains along [EF]. Measurement data refer to as-quenched and T6 condition, simulation consider the asquenched condition.

temperature, natural convection takes place with all cooling curves tending towards the same value. Temperature is almost uniform after ca. 650 seconds so that internal stresses due to thermal gradients will no longer evolve.

\subsection{Residual strain measurements}

\subsubsection{Neutron diffraction}

The ND measurement technique is well suited for Al-based alloys since aluminium is rather transparent to neutrons [6].

Forging A was taken for RS measurements by ND in the T6 state, i.e. after artificial ageing. Strain measurements by ND were performed on forging B in the as-quenched and in T6 state. ND measurement in the as-quenched state was done at the SALSA diffractometer located at the Institut Laue-Langevin (ILL) in Grenoble, France. ND measurements in the T6 state were done at STRESS-SPEC diffractometer located at FRM II in Garching, Germany. In both research institutes, strain measurements in three mutually orthogonal directions (radial, axial and hoop) were performed on three radial scan lines (Fig. 2) using the (311) aluminium reflection. A detailed description of the method, especially the used wavelength and gauge volume, is given in Ref. [7].

In direction $\mathrm{i}$, the component of the elastic strain tensor, $\varepsilon_{\mathrm{ii}}$, is derived from the interplanar spacing $d_{i}$ as follows:

$\varepsilon_{\mathrm{ii}}^{311}=\left(d_{\mathrm{i}}^{311}-\mathrm{d}_{0}^{311}\right) / \mathrm{d}_{0}^{311}=-\cot \theta \Delta \theta$ where the subscript 0 refers to the absence of macroscopic stress, $2 \theta$ is the scattering angle and $2 \Delta \theta$ is the peak shift between sample and stress-free reference measurements. The reference interplanar spacing, $\mathrm{d}_{0}$, was measured on a stress-free probe material cut from the forgings. It was used after checking that chemical composition was uniform within the forgings. Stress components are calculated using the generalised Hooke's law, which writes for an isotropic material using Einstein's summation convention:

$\sigma_{\mathrm{ij}}=\frac{E}{(1+v)} \varepsilon_{\mathrm{ij}}+\frac{\nu E}{(1+v)(1-2 v)} \varepsilon_{\mathrm{kk}} \delta_{\mathrm{ij}}$

where $i, j=r, z, \theta$ indicate the components relative to chosen orthogonal axes.

Although ND stress measurements is a well established method, two sources of uncertainty in the stress calculation must be considered. Firstly, the elastic constants $\mathrm{E}$ and $v$ depend, at the atomic scale, on crystal orientation and may differ from the macroscopic constants. The diffraction peak-specific elastic constants $\mathrm{E}^{311}=70.2 \mathrm{GPa}$ and $v^{311}=$ 0.35 for texture-free aluminium were used [8]. Secondly, the trace in Hooke's law propagates any error in one strain component to all stress components. Therefore these two sources of error make stress calculations more prone to deviations than strain measurements. Nevertheless for engineering purposes stresses are more commonly shown than strains.

\subsection{Strain gauge measurements}

Strain gauge measurements by dissection method [9] have been performed on forging $\mathrm{A}$ in the T6 state. This measurement technique allows measuring surface strains and is complementary to ND which allows measuring within a part, but not at the very surface. It is also applicable and affordable in an industrial context.

Axial and hoop strains are measured by rosette strain gauges positioned on the forging surface as shown in Fig. 2.

Ten rosette strain gauges were used along the central through-hole at seven different positions (Fig. 2). Among them two rosette strain gauges oriented at $0^{\circ}$ and $90^{\circ}$ circumferential position to each other were used at each of the three positions corresponding to the extremity of the neutron scan lines (points A, C and E). Four other rosettes also oriented two by two at $0^{\circ}$ and $90^{\circ}$ were located at 2 positions at the outer surface. The forging was instrumented with the strain gauges and dissected for residual strain measurements after T6 heat treatment, i.e. after quenching and artificial ageing. Radial strains are calculated using the zero normal stress condition, i.e. $\sigma_{\mathrm{rr}}=0$ in Eq. 2 . Residual elastic strains are converted into stresses using Eq. 2 with the macroscopic elastic constants $\mathrm{E}=74 \mathrm{GPa}$ and $v=0.3$ measured for AA2618 at $298 \mathrm{~K}$ $\left(25^{\circ} \mathrm{C}\right)[7]$.

\section{Results}

\subsection{Heat flow during quenching}

In order to simulate the strongly position-dependant heat flow during quenching, four different heat flux distributions corresponding to four different surface regions of the forging were defined in the FE model. The identification procedure developed by Rappaz et al. [10] was used. It is based on the least square technique to minimise the difference between measured and simulated temperature and it includes a maximum a posteriori algorithm. Each heat flux vs temperature curve is defined by 4 points with a linear interpolation in-between performed in the FE simulation to limit the number of variables. The inverse method is first run with 3 variable heat fluxes at fixed temperatures $423 \mathrm{~K}, 523 \mathrm{~K}$ and $873 \mathrm{~K}\left(150{ }^{\circ} \mathrm{C}, 250^{\circ} \mathrm{C}\right.$ and $\left.600{ }^{\circ} \mathrm{C}\right)$ and a heat flux value of $0 \mathrm{~W} \mathrm{~m}^{-2}$ at $373 \mathrm{~K}\left(100^{\circ} \mathrm{C}\right)$. The inverse method is then run a second time with 4 variable temperatures around $373 \mathrm{~K}, 423 \mathrm{~K}, 523 \mathrm{~K}$ and $873 \mathrm{~K}\left(100{ }^{\circ} \mathrm{C}, 150{ }^{\circ} \mathrm{C}, 250{ }^{\circ} \mathrm{C}\right.$ and $\left.600{ }^{\circ} \mathrm{C}\right)$ 


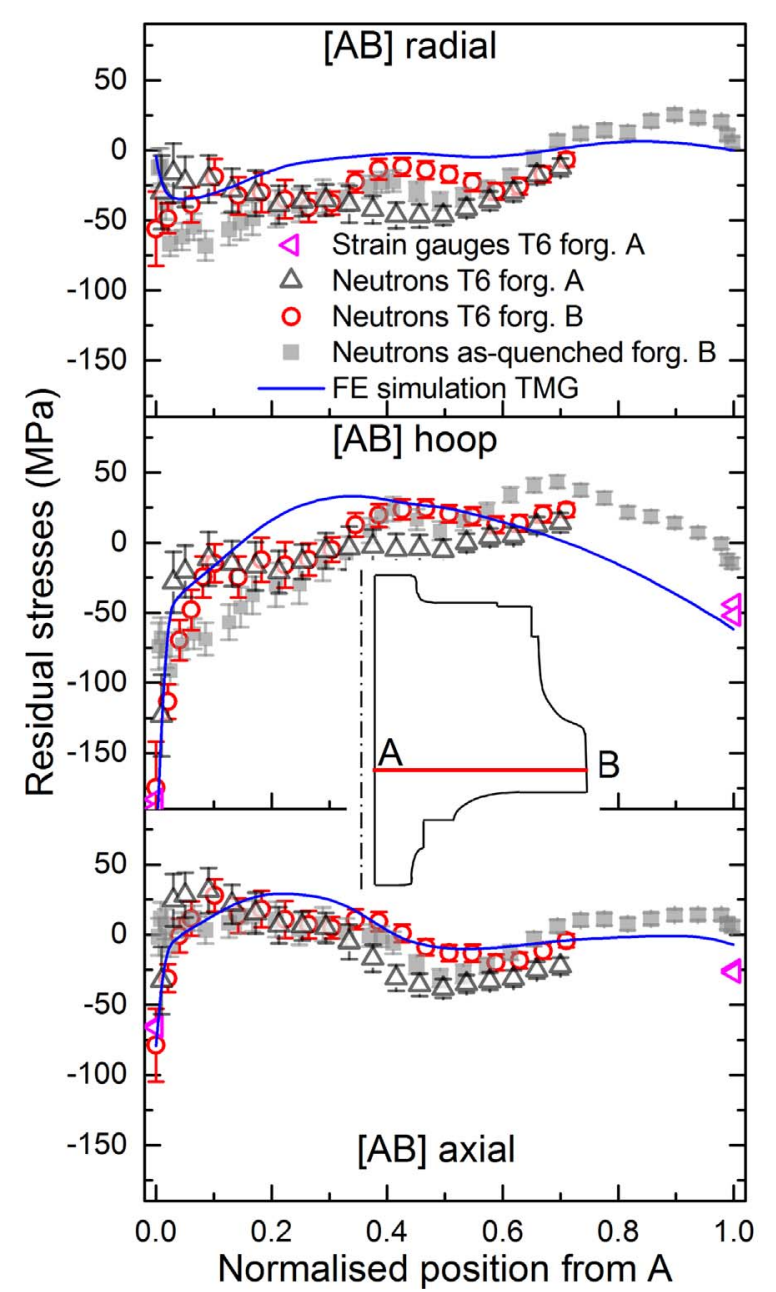

(a)

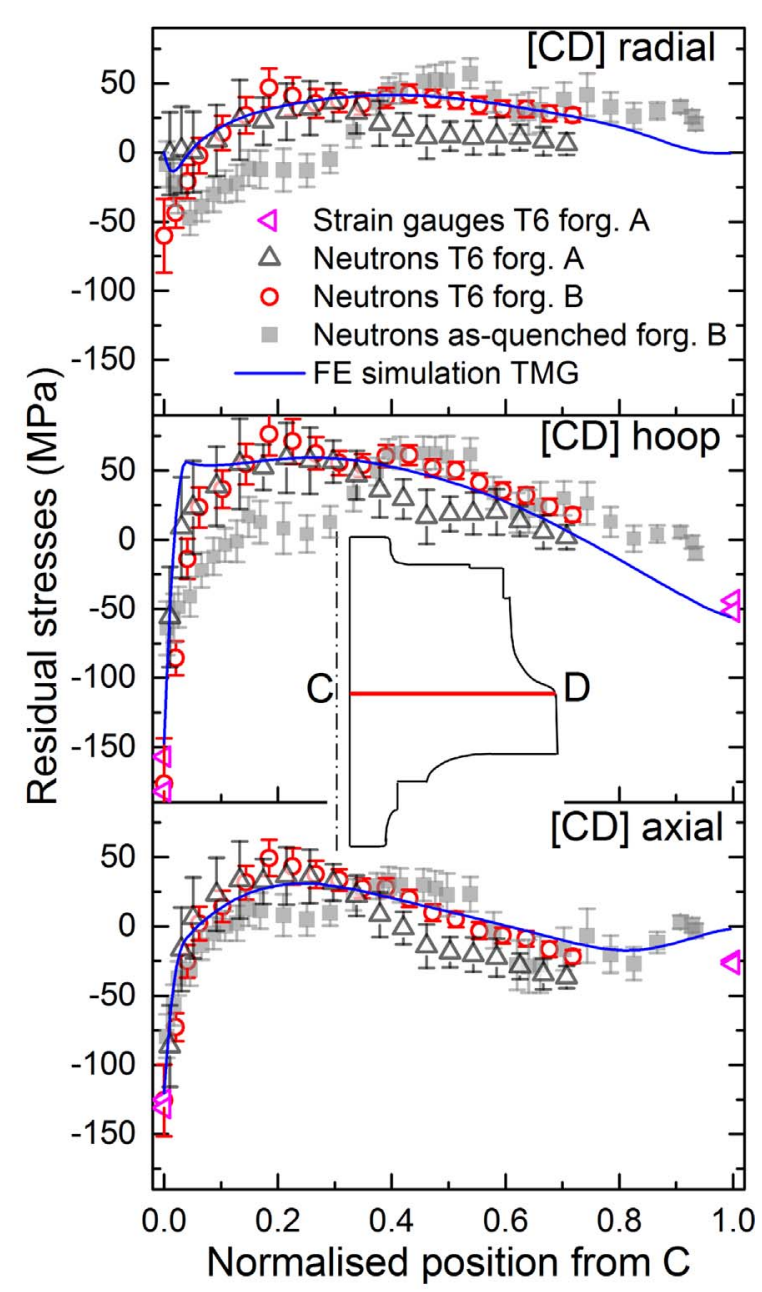

(b)

Fig. 6. Comparison between measured and simulated residual stress components (a) along [AB] and (b) [CD]. The legend in radial configurations applies for all the graphs.

using the heat fluxes identified in the first run. A final run is performed with 3 variable heat fluxes using the temperatures identified in the second run. Therefore, 4 temperature and 3 heat flux values are identified for each of the 4 surface regions defined in Fig. 3a.

The optimised heat fluxes and the measured and simulated temperature evolutions are given in Fig. 3.

Although the heat fluxes are interpolated linearly using only four values over the four surfaces, the simulated temperatures match relatively well the measured temperatures. Therefore the overall cooling behaviour of the forging is well captured. This is mandatory for a reliable stress analysis.

\subsection{Residual strains and stresses}

An uncoupled heat transfer and subsequent thermal-stress analysis is performed using the heat flux coefficients given in Fig. 3-a. The mesh is made of quadratic quadrilateral elements (DCAX8) of about $3 \mathrm{~mm}$ $\times 3 \mathrm{~mm}$ in size. It is refined in the first $10 \mathrm{~mm}$ close to the central through-hole with elements of about $1 \mathrm{~mm} \times 3 \mathrm{~mm}$ in order to capture the steep strain and stress gradients in this region. The temperature field is recorded in the Abaqus results file during the heat transfer analysis. This file is then used as input to the thermal-stress analysis of quenching. Due to the axisymmetry of the forging the stress tensor in cylindrical coordinates writes: $\sigma=\left(\begin{array}{ccc}\sigma_{r r} & \sigma_{r z} & 0 \\ \sigma_{r z} & \sigma_{z z} & 0 \\ 0 & 0 & \sigma_{\theta \theta}\end{array}\right)$

where $r, \theta$ and $z$ are the radial, hoop and axial directions respectively. Shear component $\sigma_{r z}$ is small but different from zero due to the forging shape.

Two numerical thermo-mechanical models based on an elastoviscoplastic constitutive law with additive hardening (Chaboche-type model) presented in Ref. [3] are used in the FE quenching simulations:

i) the TM (thermo-mechanical) model ignoring precipitation in which the yield strength depends only on temperature and is measured in a state as close as possible to supersaturated solid solution i.e. after a "perfect" quench,

ii) and the TMG (thermo-mechanical model based on Gleeble tests) model accounting for precipitation in a simple but realistic way. Instead of modelling precipitation that occurs during quenching, it is accounted for directly in the constitutive equation. The model parameters were identified using a limited number of tensile tests achieved after representative interrupted cooling paths in a Gleeble machine and validated against block-jaws tests.

The materials properties (thermal diffusivity, coefficient of thermal expansion, Young's modulus and Poisson's coefficient) are given in Ref. [7] and the model parameters of the TM and TMG models are given in Ref. [3]. Both models assume that the Bauschinger effect is negligible 

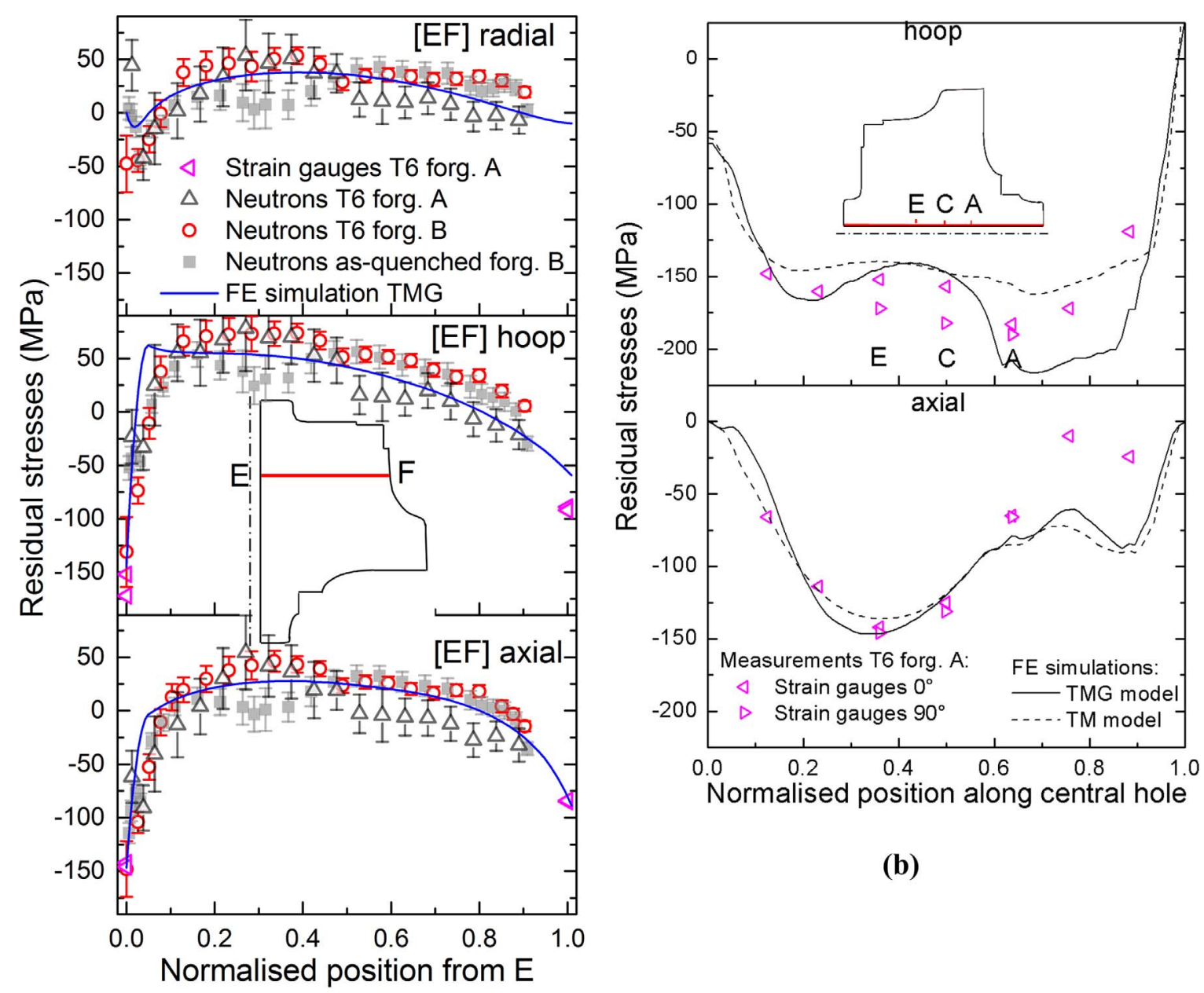

(b)

(a)

Fig. 7. Comparison between measured and simulated residual stresses (a) along [EF] and (b) along the central hole.

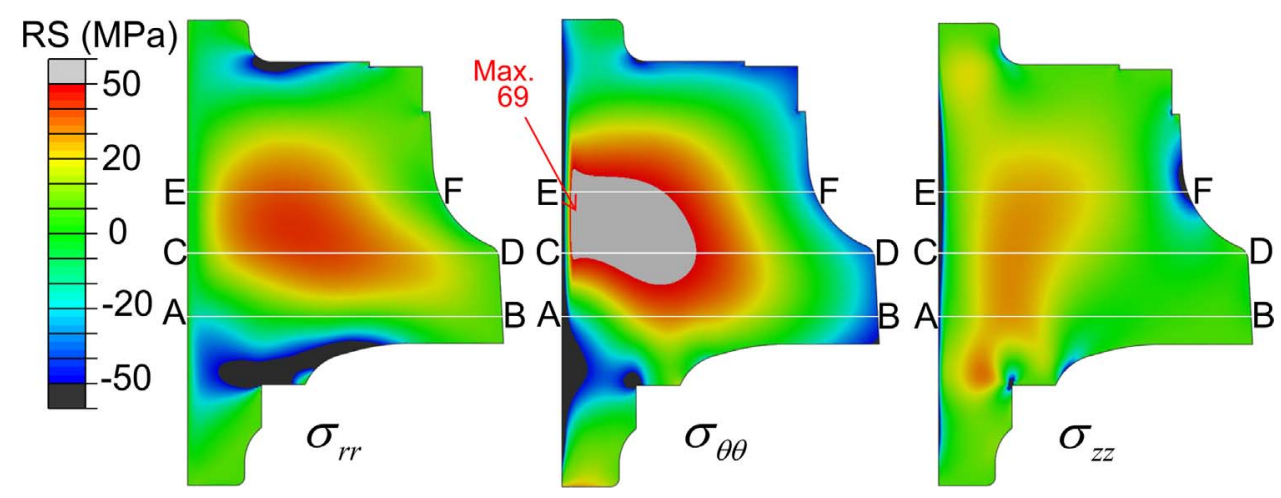

Fig. 8. Calculated residual radial, hoop and axial stress components using the TMG model.

during quenching as this is the case for water quenched plates [11]. It is also assumed that plastic strain recovery takes place above $623 \mathrm{~K}$ $\left(350{ }^{\circ} \mathrm{C}\right)$ as reported in Ref. [3].

The simulated as-quenched residual strain profiles using the TMG model are given in Figs. 4 and 5 together with the residual strains measured by ND. Residual strains measured by strain gauges in T6 state are also shown. The results predicted by the TM model are only given in terms of stresses when they significantly differ from those predicted by the TMG model.

The results in terms of residual stresses calculated using the measured strains are given in Figs. 6 and 7 along the different scan lines.

\section{Discussion}

\subsection{RS in forgings with a central hole}

The residual strain profiles in T6 state measured in forgings A and B are similar, thus indicating the good reproducibility of the quench. The measured residual strain profiles in as-quenched and in T6 state are also very close to each other. This means that the ageing of AA2618 to T6 state does not reduce the strains and stresses induced during 


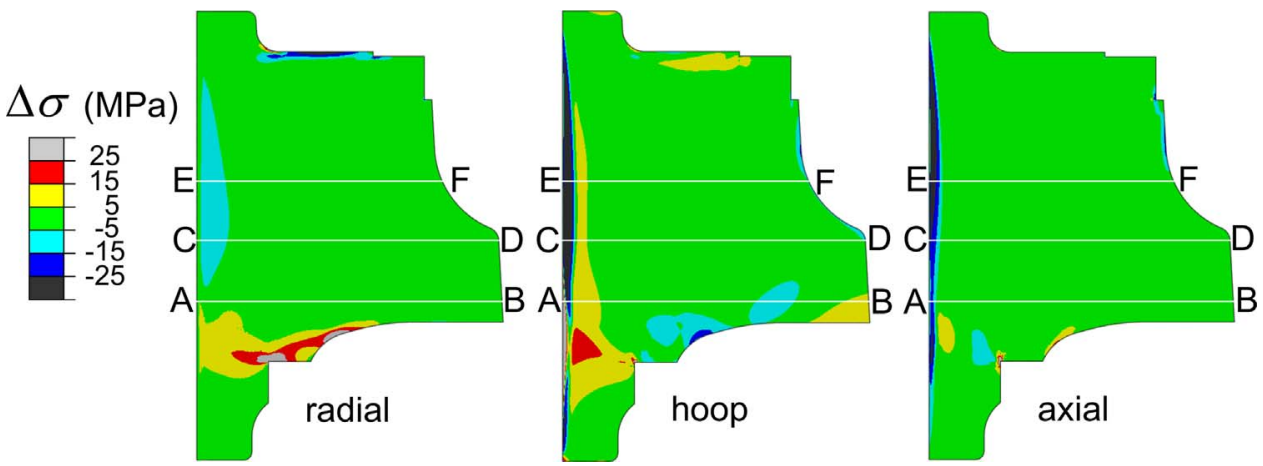

Fig. 9. Subtraction of stresses predicted by the TM model (ignoring precipitation) to those predicted by the TMG model in forging with a central hole.
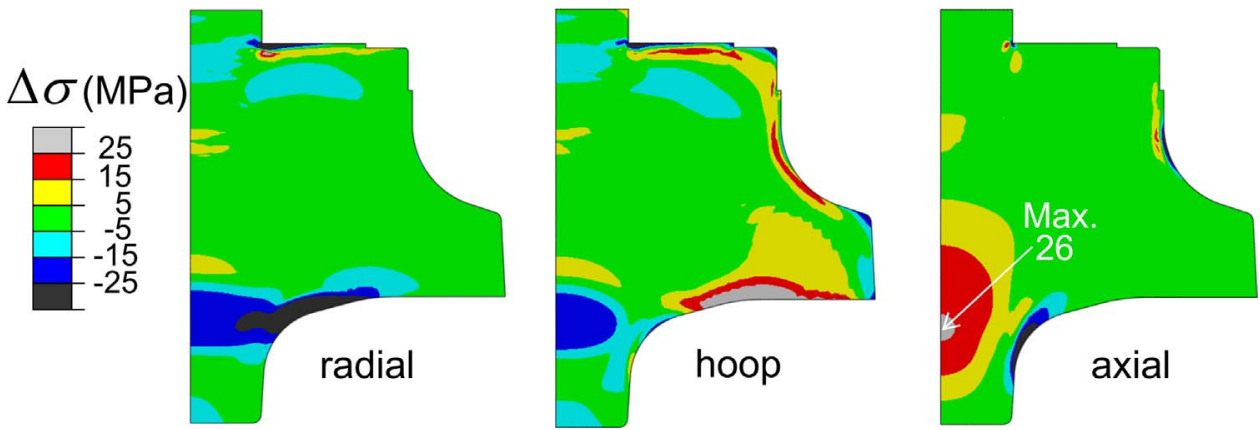

Fig. 10. Subtraction of stresses predicted by the TM model (ignoring precipitation) to those predicted by the TMG model in forging without central hole. Adapted from Ref. [7].

quenching. This is expected for two reasons: The rather low temperature of the T6 treatment and the high thermal stability of the AA2618 alloy [1].

The neutron measurements together with the surface measurements by strain gauges are well reproduced by the $\mathrm{FE}$ quenching simulation. The different error bars on the ND results are due to different measurement statistics because of different gauge volumes and counting times. The good agreement between measurements and simulation is due to the quality of both the calculated thermal field evolution and the material model.

Figs. 6 and 7 show that the FE quenching simulation using the TMG model predicts relatively well the measured RS. It is worth to keep in mind, that since all stress components are dependent on all measured strain components by Hooke's law, uncertainties in one strain direction, for example due to long penetration paths and thus lower measurement statistics, are visible in all three stress directions.

The as-quenched residual stress distributions simulated with the TMG model are shown in Fig. 8.

As already shown by the measurements, the forging is in a bi-axial compression state close to the surfaces and in a tri-axial tension state at the center. The maximal tensile RS is found close to $\mathrm{E}$ (i.e. close to the central hole) for the hoop component as indicated in Fig. 8. The accurate knowledge of those stresses is important for the simulation of further processing steps such as machining during which as-quenched residual stresses are partially relaxed.

The results predicted by the TM model are very close to those predicted by the TMG model as shown by the difference plots in Fig. 9 where the regions in green indicate that both models predict the same RS with a difference of $\pm 5 \mathrm{MPa}$. These differences were more pronounced in water quenched thick AA7xxx plates [10] and they were attributed to surface precipitation hardening by cluster formation during quenching. The low difference on the forging between RS predicted by the TM and the TMG model indicates that precipitation actually occurs in the forgings with a central hole, but has a relatively small impact on RS.

The largest differences are localised along the central hole where the TMG model predicts higher RS in absolute value than those predicted by the TM model. This region being in compression, the differences between the predictions of the two models are less critical than the differences in the regions in tension. This means that for the forging with central hole, ignoring the impact of possible precipitation on the yield strength in the material model is acceptable.

\section{2. $R S$ in forgings}

To determine the limit of applicability of the TM model, the same methodology was applied to a forging without central hole with an outer diameter close to the one of the forging with central hole [4]. The results in terms of difference plots between the TM model and the TMG model are shown in Fig. 10. Compared to the difference plots for the forging with central hole (Fig. 9), the differences between TM and TMG models are more pronounced for the forging without central hole (Fig. 10), especially at the position of the maximal difference of $26 \mathrm{MPa}$ for the axial component (in tension) where the TMG model predicts axial RS (Max. $126 \mathrm{MPa}$ ) around 26\% higher than those predicted by the TM model (Max. $100 \mathrm{MPa}$ ).

This means that contrary to the forging with central hole, the forging without central hole and around $0.5 \mathrm{~m}$ in diameter is significantly affected by precipitation during quenching owing to its large massiveness and thus by its impact on the yield strength and on the residual stresses. Although the TMG model is not required for the forging with a central hole due to its relative fast cooling, it becomes necessary for reliable RS prediction in the forging without central hole due to its higher cross section.

\section{Conclusion}

A material model calibrated using interrupted quench tests and validated upon constrained cooling is used in a FE model to simulate the stress build-up during quenching of large AA2618 forgings. Prior to the stress analysis the thermal field evolution in the forging is carefully characterized by using temperature and position dependent heat transfer conditions. The residual strain profiles in as-quenched and T6 states measured at the two neutron diffractometers SALSA and 
STRESS-SPEC are very similar meaning that the T6 peak-aged treatment does not affect the internal stresses. These neutron measurements together with surface measurements by strain gauges are well reproduced by the $\mathrm{FE}$ quenching simulations using the adequate material model. In the forging with a central hole, ignoring the impact of possible precipitation during quench on the yield strength in the material model is acceptable. On the other hand, in the forging of similar size but with no central hole, considering the impact of precipitation during quench on the yield strength is required.

\section{Acknowledgements}

This work is funded by the Competence Center for Materials Science and Technology (http://www.ccmx.ch/) in the frame of the project entitled "Measurements and modelling of residual stress during quenching of thick heat treatable aluminium components in relation to their microstructure" involving EPF Lausanne, PSI Villigen, Univ. de Bretagne Sud Lorient, Constellium CRV and ABB Turbo Systems Ltd. The authors are indebted to W. Erb (ABB Turbo Systems Ltd) for thermocouple and strain gauge instrumentation of the forging and the corresponding measurements. The authors are grateful to the German neutron reactor FRM II for providing beam time at STRESS-SPEC and to the international neutron source at ILL for providing beam time at
SALSA.

\section{References}

[1] G. Pouget, C. Sigli, Mater. Sci. Forum 794 (2014) 691-696.

[2] J.R. Davis, G.M. Davidson, S.R. Lampman, Heat Treating of Aluminum 4, ASM International, Materials Park, Ohio, 1995, pp. 841-879.

[3] N. Chobaut, D. Carron, P. Saelzle, J.-M. Drezet, Metall. Mater. Trans. A 47 (2016) 5641-5649.

[4] N. Chobaut, P. Saelzle, G. Michel, D. Carron, J.-M. Drezet, JOM 67 (2015) 984-990.

[5] D. Büche, N. Hofmann, and P. Sälzle, Paper Presented at the NAFEMS World Congress, 2005.

[6] J.M. Drezet, A. Phillion, Metall. Mater. Trans. A 41 (2010) 3396-3404.

[7] N. Chobaut, EPFL (Ph.D. thesis) nº559, DOI:http://dx.doi.org/10.5075/epflthesis-6559 〈http://infoscience.epfl.ch/record/205768/files/EPFL_TH6559.pdf〉, 2015.

[8] M.T. Hutchings, P.J. Withers, T.M. Holden, T. Lorentzen, Introduction to the Characterization of Residual Stress by Neutron Diffraction, CRC Press, 6000 Broken Sound Pkwy NW, Boca Raton, Florida, 2005, p. 213.

[9] N. Tebedge, G. Alpsten, L. Tall, Exp. Mech. 13 (1973) 88-96.

[10] M. Rappaz, J.-L. Desbiolles, J.-M. Drezet, C.-A. Gandin, A. Jacot, P. Thévoz, Modelling of Casting, Welding and Advanced Solidification Processes MCWASP, TMS, London, 1995, pp. 449-457.

[11] N. Chobaut, D. Carron, S. Arsène, P. Schloth, J.M. Drezet, J. Mater. Process. Technol. 222 (2015) 373-380. 